\title{
Microwave reflection and transmission characteristics through plasma slab based on PLRC
}

\author{
Jinzu Ji, Yunpeng Ma* (Corresponding Author) \\ School of Aeronautic Science and Engineering, Beihang University, Beijing, China \\ e-mail: jijinzu@buaa.edu.cn,e-mail: jijinzu@buaa.edu.cn
}

\begin{abstract}
Keywords:Computational electromagnetics; Piecewise linear recursive convolution (PLRC); Finite-difference time-domain (FDTD); Plasma; Reflection coefficient; Transmission coefficient
\end{abstract}

\begin{abstract}
PLRC)is proposed for cold plasma medium using finite-difference time-domain (FDTD) method. In PLRC algorithm, the electric field with time is piecewise interpolated and the recursive convolution was introduced. The reflection and transmission coefficients were calculated by the electromagnetic response of a differential Gaussian pulse. The calculation results are validated with analytic values and the results agree well with each other. The calculation results show that higher frequency microwave can transmit through the plasma and lower frequency is forbidden.
\end{abstract}

\section{Introduction}

Finite-difference time-domain (FDTD) method has been widely used to model the interaction of electromagnetic wave with non-dispersive materials since Yee introduced the Yee's scheme in 1966 [1]. In Yee's algorithm, the dielectric and conductive medium can be calculated very well, but it cannot be calculated directly for dispersive medium, such as cold plasma.In order to simulate electromagnetic phenomena in frequency-dependent media, several approaches have been proposed. The strategy is to use the inverse Fourier transform by which the frequency-dependent constitutive relation is transformed to the time-domain. These techniques include the recursive convolution (RC)[2], piecewise linear recursive convolution (PLRC) [3] and auxiliary differential equation (ADE)[4]. By mapping the frequency-domain relation is to Z-domain, then the Z transform (ZT) technique is developed[5, 6]. Recently, exponential time differential (ETD) algorithm is proposed to raise the accuracy[7, 8]. The RC approaches have typically been faster and have required fewer computer memory resources than the ADE and ZT approaches, but the latter two have usually been more accurate. In addition, it has usually been easier to treat the case of a medium with multiple poles in the susceptibility function using the RC methods[9]. The PLRC method to be used in this paper, however, provides accuracy that rivals that of the existing ADE and ZT approaches, yet retains the speed, memory and multiple pole advantages of the RC approach.

The PLRC scheme is utilized to study the plasma slab's reflection and transmission coefficients in this paper.The algorithm isvalidated by comparing the analytical results. The characteristics of the microwave interaction with the plasma slab are analyzed such as frequency's influence on the reflection and transmission coefficients.

Time factor of $e^{j \omega t}$ is assumed and suppressed throughout this paper.

\section{Formulation}

In a linear dispersive medium, the electric flux density is related to the electric field intensity by

$$
D(t)=\varepsilon_{0} \varepsilon_{\infty} E(t)+\varepsilon_{0} \int_{0}^{t} E(t-\tau) \chi(\tau) \mathrm{d} \tau \text {.(1) }
$$

Using the notation $D^{n}=D(n \Delta t)$ and $E^{n}=E(n \Delta t)$, (1) can be written in discrete form as

$$
D^{n}=\varepsilon_{0} \varepsilon_{\infty} E^{n}+\varepsilon_{0} \int_{0}^{n \Delta t} E(n \Delta t-\tau) \chi(\tau) \mathrm{d} \tau(2)
$$

where $\varepsilon_{0}$ is the permittivity of free space, $\varepsilon_{\infty}$ is the dielectric constant of the medium at infinite frequency, and $\chi(t)$ is the susceptibility function of the medium. For cold plasma, $\varepsilon_{\infty}=1$. 
A piecewise linear approximation to the continuous time electric field $E(t)$ over a given interval $[i \Delta t,(i+1) \Delta t]$ can be expressed in terms of the discrete time values $E^{i}$ and $E^{i+1}$ as

$$
E(t)=E^{i}+\frac{E^{i+1}-E^{i}}{\Delta t}(t-i \Delta t)
$$

The piecewise linear time-reversed and offset form of the electric field that appears in the convolution in (2) is given by

$$
E(n \Delta t-\tau)=E^{n-m}+\frac{E^{n-m-1}-E^{n-m}}{\Delta t}(\tau-m \Delta t)
$$

Substitution of (4) into (2) yields

$$
\begin{aligned}
& D^{n}=\varepsilon_{0} \varepsilon_{\infty} E^{n}+ \\
& \varepsilon_{0} \sum_{m=0}^{n-1}\left[E^{n-m} \chi^{m}+\left(E^{n-m-1}-E^{n-m}\right) \xi^{m}\right]
\end{aligned}
$$

where

$$
\begin{aligned}
& \chi^{m}=\int_{m \Delta t}^{m \Delta t+\tau} \chi(\tau) \mathrm{d} \tau \\
& \xi^{m}=\frac{1}{\Delta t} \int_{m \Delta t}^{m \Delta t+\tau}(\tau-m \Delta t) \xi(\tau) \mathrm{d} \tau
\end{aligned} .
$$

After substituting the expression for $D^{n}$ and $D^{n+1}$, the general form of the FDTD update equation for the electric field becomes

$$
\begin{aligned}
& E^{n+1}=\frac{\varepsilon_{\infty}-\xi^{0}}{\varepsilon_{\infty}-\xi^{0}+\chi^{0}} E^{n}+\frac{1}{\varepsilon_{\infty}-\xi^{0}+\chi^{0}} \psi^{n}+ \\
& \frac{\Delta t}{\varepsilon_{0}\left(\varepsilon_{\infty}-\xi^{0}+\chi^{0}\right)} \nabla \times H^{n+1 / 2}
\end{aligned}
$$

where $\Delta \chi^{m}=\chi^{m}-\chi^{m+1}, \Delta \xi^{m}=\xi^{m}-\xi^{m+1}$ and

$$
\psi^{n}=\sum_{m=0}^{n-1}\left[E^{n-m} \chi^{m}+\left(E^{n-m-1}-E^{n-m}\right) \chi^{m}\right] .
$$

A recursion relation for the quantity $\psi^{n}$ can be derived for Debye, cold plasma and Lorentz media and is of the form

$$
\psi^{n}=\left(\Delta \chi^{0}-\Delta \xi^{0}\right) E^{n}+\Delta \xi^{0} E^{n-1}+C_{\text {ree }} \psi^{n-1}
$$

It will be shown that the constants $\Delta \chi^{0}, \Delta \xi^{0}$, and $C_{\text {rec }}$ are real value for cold plasma.

To use the PLRC algorithm, the quantities $\chi^{m}, \Delta \chi^{m}, \xi^{m}, \Delta \xi^{m}$ and $C_{\text {rec }}$, all of which depend on the susceptibility function of the medium, must be calculated. For cold plasma, $\varepsilon_{\infty}=1$ and the frequency domain susceptibility function is given by

$$
\chi(\omega)=\frac{\omega_{p}^{2}}{j \omega\left(j \omega+v_{c}\right)}=\frac{\omega_{p}^{2}}{v_{c}}\left(\frac{1}{j \omega}-\frac{1}{j \omega+v_{c}}\right)
$$

where $\omega_{p}$ is plasma's frequency, $v_{c}$ is collision frequency, $\omega$ is angular frequency of electromagnetic wave, $j=\sqrt{-1} . \omega_{p}$ is defined by $\omega_{p}^{2}=n_{e} e^{2} / \varepsilon_{0} m_{e}$, where $n_{e}$ is electron's density, $e$ is electron's charge and $m_{e}$ is electron's mass. The time domain susceptibility function obtained by computing the inverse Fourier transform of $\chi(\omega)$ is

$$
\chi(t)=\frac{\omega_{p}^{2}}{v_{c}}\left(1-e^{-v_{c} t}\right) u(t)
$$

where $u(t)$ is the unit step function. Constants for recursive convolution are 


$$
\begin{aligned}
& \chi^{0}=\frac{\omega_{p}^{2}}{v_{c}}\left(\Delta t-\frac{1}{v_{c}}\left(1-e^{-v \Delta t}\right)\right) \\
& \xi^{0}=\frac{\omega_{p}^{2}}{v_{c}}\left(\frac{\Delta t}{2}-\frac{1}{v_{c}}\left(1-\left(1+v_{c}\right) e^{-v \Delta t}\right)\right) \\
& \Delta \chi^{0}=-\frac{\omega_{p}^{2}}{v_{c}^{2}}\left(1-e^{-v \Delta t}\right)^{2} \\
& \Delta \xi^{0}=-\frac{\omega_{p}^{2}}{v_{c}^{2}}\left(1-\left(1+v_{c}\right) e^{-v \Delta t}\right)\left(1-e^{-v \Delta t}\right) \\
& C_{\text {rec }}=e^{-v \Delta t}
\end{aligned}
$$

\section{Numerical validation}

The PLRC method was applied to the problem of computing the reflection and transmission coefficient for a plane wave pulse normally incident on a cold plasma slab. This simple example in one dimension was chosen because exact analytical solutions are available to which the computed quantities can be compared.

The wave is normally incident on a plasma slab with a thickness of $15 \mathrm{~mm}$. The incident waves are differential Gaussian pulse which is expressed as

$$
f(t)=\left(\frac{t-t_{0}}{T}\right) e^{-4 \pi\left(\frac{t-t_{0}}{T}\right)^{2}}
$$

where $T=10 \mathrm{ps}$. The pulse's frequency spectrum peaks at $50 \mathrm{GHz}$ and is $10 \mathrm{~dB}$ down from the peak at $100 \mathrm{GHz}$ and can simulate microwave band's behavior. The plasma parameters are as follows: $\omega_{p}=2 \pi \times 28.7 \times 10^{9} \mathrm{rad} / \mathrm{s}, \quad v=20 \times 10^{9} \mathrm{rad} / \mathrm{s}$. The solution space consists of 800 cells and each cell is $75 \mu \mathrm{m}$ long. The time step is 0.125 ps. The plasma occupies cells $300-500$ and the cells $0-300$ and 500-800 are free space. To prevent the spurious reflections from the truncated boundaries, both ends of the solution space are applied by the Mur's absorbing boundary conditions (ABC). The total iterative number is 800 times. The reflection and transmission coefficients are computed from the reflected and transmitted pulses through discrete Fourier transform of the time dependent electric field components.

Figure 1 shows the dependence of the reflection coefficient of the plasma slab on microwave frequency $1 \mathrm{GHz} 100 \mathrm{GHz}$. The dependence of the transmission coefficient of the plasma on frequency is depicted in Figure 2. It can be seen that the simulation results coincide with analytical values very well. The phase of reflection and transmission coefficients are also calculated and illustrated in Figure 3 and Figure 4.These figures exhibit that the PLRC algorithm is very accurate. The RC simulation results are also illustrated in these figures and the comparison show that PLRC has more accuracy.

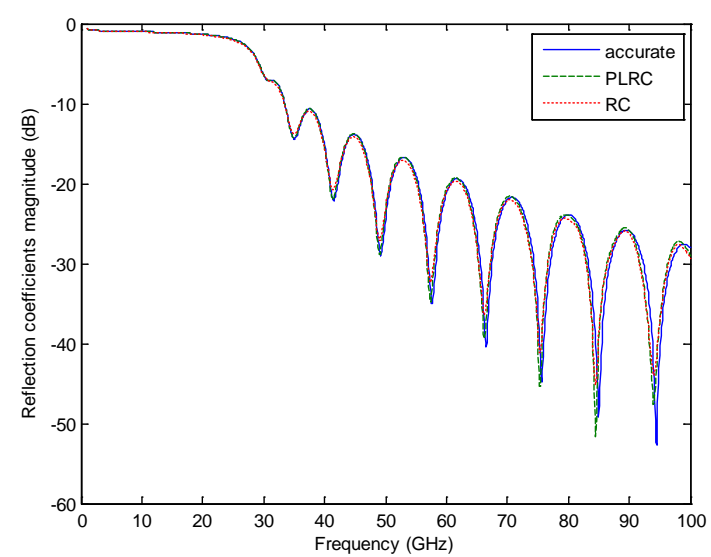

Figure 1 Dependence of the reflection coefficient of the isotropic plasma slab on frequency 


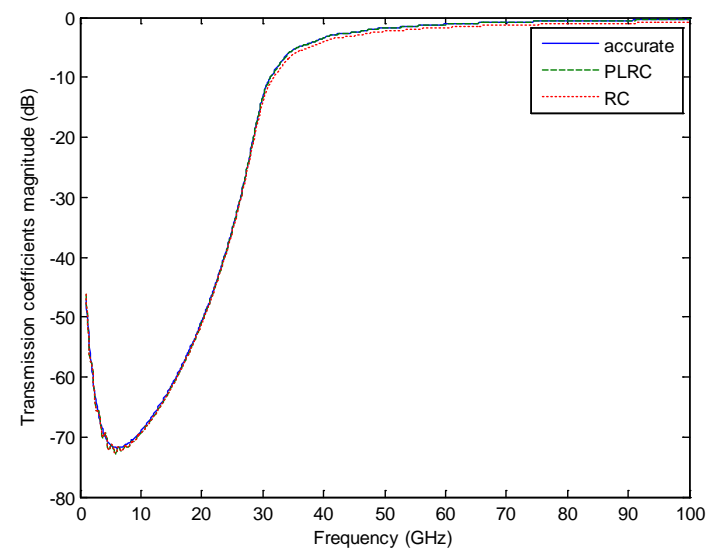

Figure 2 Dependence of the transmission coefficient of the isotropic plasma slab on frequency

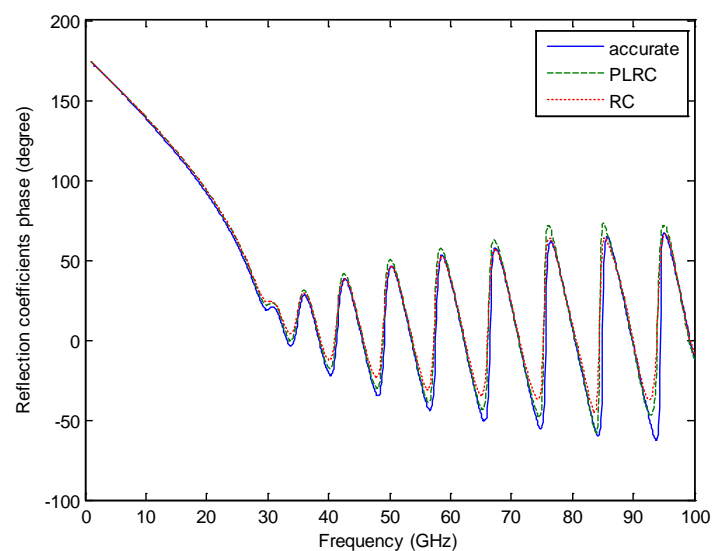

Figure 3 Dependence of the reflection coefficient's phase of the isotropic plasma slab on frequency

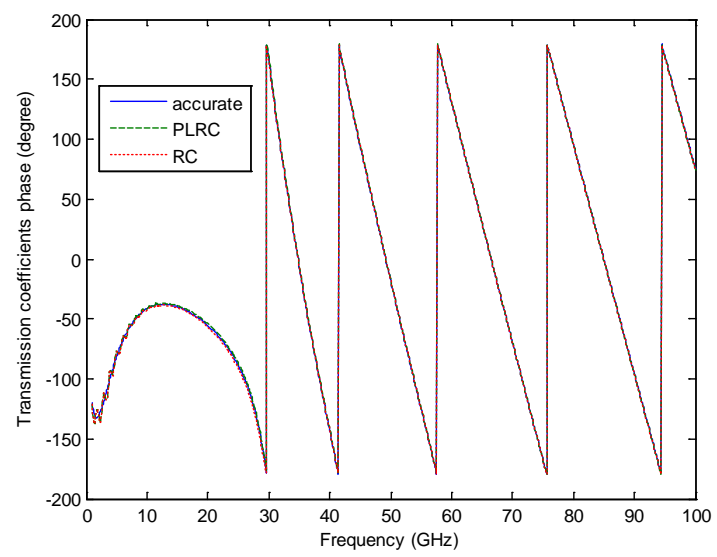

Figure 4 Dependence of the transmission coefficient's phase of the isotropic plasma slab on frequency

From Figure 1, most of the incident energy is reflected at lower frequency, especially below plasma's frequency, because the incident frequency is smaller than plasma's and the wave is evanescent in the slabwhich results wave's forbidden. As the frequency ascends, the reflection coefficients get lower with resonance. The transmission coefficient gets higher as the frequency ascends.

\section{Conclusion}

In this article, we have presented PLRC scheme for electromagnetic wave propagation in 
plasmas. To verify the PLRC algorithm, we have computed the reflection and transmission coefficients of transient electromagnetic wave through a plasma slab. The simulation results demonstrate the effectiveness and accuracy of the algorithm. The numerical results are in excellent agreement with analytical values. The comparison of the RC and PLRC results shows that the PLRC is more accuracy than RC. The characteristics of the reflection and transmission are also analyzed and the results show that at lower frequency, most of the energy is reflected and at higher frequency, most of the energy is transmitted.

\section{References}

[1] K. S. Yee, "Numerical solution of initial boundary value problems involving maxwell's equations in isotropic media," Antennas and Propagation, IEEE Transactions on, vol. 14, pp. 302-307, 1966.

[2] R. Luebbers, F. P. Hunsberger, K. S. Kunz, R. B. Standler, and M. Schneider, "A frequency-dependent finite-difference time-domain formulation for dispersive materials," Electromagnetic Compatibility, IEEE Transactions on, vol. 32, pp. 222-227, 1990.

[3] D. F. Kelley and R. J. Luebbers, "Piecewise linear recursive convolution for dispersive media using FDTD," Antennas and Propagation, IEEE Transactions on, vol. 44, pp. 792-797, 1996.

[4] O. P. Gandhi, B. Q. Gao, and J.-Y. Chen, "A frequency-dependent finite-difference time-domain formulation for general dispersive media," Microwave Theory and Techniques, IEEE Transactions on, vol. 41, pp. 658-665, 1993.

[5] D. M. Sullivan, "Frequency-dependent FDTD methods using Z transforms," Antennas and Propagation, IEEE Transactions on, vol. 40, pp. 1223-1230, 1992.

[6] D. M. Sullivan, "Z-transform theory and the FDTD method," Antennas and Propagation, IEEE Transactions on, vol. 44, pp. 28-34, 1996.

[7] Y. Liu and H. W. Yang, "Shift operator method and Runge-Kutta exponential time differencing method for plasma," Optik, vol. 122, pp. 2086-2089, 20112011.

[8] X. Zhuansun, X. Ma, and Q. Liu, "An Exponential Time Differencing Algorithm for the FDTD-PML Analysis of Nonlinear Photonic Bandgap Structures," Ieee Transactions on Magnetics, vol. 50, Feb 2014.

[9] R. J. Luebbers and F. Hunsberger, "FDTD for Nth-order dispersive media," Antennas and Propagation, IEEE Transactions on, vol. 40, pp. 1297-1301, 1992. 